Revue internationale P.M.E.

Économie et gestion de la petite et moyenne entreprise

\title{
Le rôle du repreneur dans le processus de formation de la stratégie de l'entreprise acquise
}

\section{Frédérique GRAZZINI, Jean-Pierre BOISSIN et Bertrand MALSCH}

Volume 22, numéro 3-4, 2009

URI : https://id.erudit.org/iderudit/044131ar

DOI : https://doi.org/10.7202/044131ar

Aller au sommaire du numéro

Éditeur(s)

Presses de l'Université du Québec

ISSN

0776-5436 (imprimé)

1918-9699 (numérique)

Découvrir la revue

Citer cet article

GRAZZINI, F., BOISSIN, J.-P. \& MALSCH, B. (2009). Le rôle du repreneur dans le processus de formation de la stratégie de l'entreprise acquise. Revue internationale P.M.E., 22(3-4), 139-164. https://doi.org/10.7202/044131ar
Résumé de l'article

À une époque où l'évolution démographique fait de la reprise d'entreprise un des enjeux majeurs pour les PME, ce travail de recherche suggère de prendre en compte des éléments autres que les seuls aspects techniques du processus (juridiques, fiscaux ou financiers). Il semble en effet nécessaire d'envisager certains aspects d'ordre managérial et plus particulièrement ceux en lien avec le processus de formation de la stratégie au sein de l'entreprise récemment acquise. Ainsi, l'objectif de cet article est d'éclairer les principaux liens, interactions et éventuelles interférences qui peuvent se produire entre la personne du repreneur et le processus de formation de la stratégie de l'entreprise. Cette recherche s'appuie sur une analyse de la littérature combinée à une étude de cas longitudinale menée sur une période de deux ans. Les recommandations formulées à l'issue de ce travail invitent les différentes parties concernées, dans le cadre d'une approche personnalisée, à évaluer et à analyser avec attention le niveau d'adéquation qui existe entre la personne du repreneur et la fonction de direction qu'il souhaite occuper au sein d'une entreprise spécifique.
Ce document est protégé par la loi sur le droit d'auteur. L'utilisation des services d’Érudit (y compris la reproduction) est assujettie à sa politique d'utilisation que vous pouvez consulter en ligne.

https://apropos.erudit.org/fr/usagers/politique-dutilisation/ 


\title{
Le rôle du repreneur dans le processus de formation de la stratégie de l'entreprise acquise
}

\author{
Frédérique GRAZZINI \\ Jean-Pierre BOISSIN \\ Université Pierre-Mendès-France
}

Bertrand MALSCH

Université Laval

MOTS CLÉS

\section{Transmission - Formation de la stratégie - Dirigeant Vision stratégique - Socialisation organisationnelle}

\begin{abstract}
LES AUTEURS
FRÉDÉRIQUE GRAZZINI est allocataire de recherche au CERAG (Grenoble). Ses travaux de recherche portent sur la participation des managers au processus de formation de la stratégie de leur entreprise plus particulièrement dans le cadre des approches proposées par les perspectives de la pratique en stratégie. Adresse: Université Pierre-Mendès-France, CERAG (UMR 5820 CNRS-UPMF), IAE de Grenoble, 150, rue de la Chimie, B.P. 47, 38040 Grenoble, Cédex 9, France. Courriel:<frederique.grazzini@upmf-grenoble.fr>.

JEAN-PIERRE BOISSIN est professeur à l'Université Pierre-Mendès-France (IAE de Grenoble) en management stratégique et entrepreneuriat. II a fondé et dirigé la Maison de l'entrepreneuriat de Grenoble Universités et il est actuellement directeur du Centre d'études et de recherches appliquées à la gestion (CERAG, Université Pierre-Mendès-France, UMR CNRS 5820, Grenoble). Ses travaux de recherche portent sur la structuration du management stratégique à partir d'outils bibliométriques. II développe actuellement un observatoire international de I'intention entrepreneuriale <OIE-CERAG.fr> et dirige différents travaux sur les stratégies de croissance et le gouvernement d'entreprise. Adresse: Université Pierre-Mendès-France, CERAG (UMR 5820 CNRS-UPMF), IAE de Grenoble, 150, rue de la Chimie, B.P. 47, 38040 Grenoble, Cédex 9, France. Courriel : <Jean-pierre.boissin@upmf-grenoble.fr>.

BERTRAND MALSCH est chargé d'enseignement et doctorant à l'École de comptabilité de l'Université Laval. Ses travaux de recherche se situent dans le paradigme de la recherche interprétative et visent à mettre en évidence les enjeux de pouvoir et de reproduction sociale dans une perspective socio-organisationnelle. Adresse: Faculté des sciences de l'administration, Pavillon Palasis-Prince, 2325, rue de la Terrasse, Université Laval, Québec, Québec, G1V 0A6. Courriel : <bertrand.malsch@fsa.ulaval.ca>.
\end{abstract}




\title{
RÉSUMÉ
}

À une époque où l'évolution démographique fait de la reprise d'entreprise un des enjeux majeurs pour les PME, ce travail de recherche suggère de prendre en compte des éléments autres que les seuls aspects techniques du processus (juridiques, fiscaux ou financiers). II semble en effet nécessaire d'envisager certains aspects d'ordre managérial et plus particulièrement ceux en lien avec le processus de formation de la stratégie au sein de l'entreprise récemment acquise. Ainsi, l'objectif de cet article est d'éclairer les principaux liens, interactions et éventuelles interférences qui peuvent se produire entre la personne du repreneur et le processus de formation de la stratégie de l'entreprise. Cette recherche s'appuie sur une analyse de la littérature combinée à une étude de cas longitudinale menée sur une période de deux ans. Les recommandations formulées à l'issue de ce travail invitent les différentes parties concernées, dans le cadre d'une approche personnalisée, à évaluer et à analyser avec attention le niveau d'adéquation qui existe entre la personne du repreneur et la fonction de direction qu'il souhaite occuper au sein d'une entreprise spécifique.

\begin{abstract}
At a time when demographic evolution has made takeovers one of the major concerns for SMEs, it is important to take into account different aspects beyond the merely technical (legal, tax, or financial). It would be fruitful to consider the managerial perspective, particularly in relation to the strategy making process of the recently acquired enterprise. Thus, this article highlights the main links, interactions, and possible interferences that may occur between the personality of the ownermanager and the strategy making process. This research combines insights from existing literature and observations drawn from a two-year longitudinal case study. To conclude, we suggest paying closer attention to the level of adequacy or the "fit" (Kotter, 1982), between the personality of the buyer and the managerial position he plans to occupy within the enterprise.
\end{abstract}

\section{RESUMEN}

En un tiempo en el que la evolución demográfica ha hecho de las adquisiciones uno de los retos más importantes para las PyMEs, este análisis sugiere tomar en cuenta factores distintos de los meros aspectos técnicos del proceso (legal, fiscal o financiero). Ciertamente, podría ser beneficioso considerar ciertos aspectos desde una perspectiva de gestión, especialmente los relacionados con el proceso de formación de la estrategia de la empresa recientemente adquirida. Así, el objetivo de este análisis es resaltar los vínculos principales, las interacciones y las posibles interferencias que pueden producirse entre la persona del comprador y el proceso de formación de la estrategia de la empresa. Esta investigación combina ideas tomadas de la literatura existente y de las observaciones extraídas de un estudio exhaustivo de un caso de dos años de duración. Como conclusión, sugerimos prestar mucha atención al nivel de adecuación, en otras palabras, al «ajuste » (Kotter, 1982), entre la persona del comprador y la función de gestión que éste quiere desempeñar en una empresa específica. 


\section{ZUSAMMENFASSUNG}

Auch wegen der demografischen Entwicklung der Bevölkerung ist die Nachfolgeregelung bei KMU ein zentrales Thema kommender Jahre. Vorliegender Bericht empfiehlt, neben juristischen, steuerlichen oder finanziellen Aspekten andere Element zu betrachten. So sind Fragen der Unternehmensführung, insbesondere die Strategieentwicklung des übernommenen Betriebes, zu thematisieren. Es wird auf die möglichen Verbindungen, Interaktionen und Störungen zwischen dem Nachfolger und der Strategieentwicklung eingegangen. Die Studie stützt sich auf eine eingehende Literaturanalyse und eine Fallstudie über zwei Jahre. Die formulierten Empfehlungen richten sich an die verschiedenen Anspruchsgruppen im Nachfolgeprozess und schlagen insbesondere vor, die Übereinstimmung des Nachfolgers mit der neu zu übernehmenden Führungsfunktion, zu prüfen.

\section{Introduction}

À une époque où l'évolution démographique commence déjà à entraîner un nombre important de départs à la retraite de dirigeants de PME/TPE, la reprise d'entreprise par des personnes physiques (RPP) (Deschamps, 2000), dont le nombre est estimé à 700000 d'ici 10 ans, représente un enjeu économique et social majeur (OSÉO, 2005). Malgré de nombreux dispositifs d'accompagnement, le taux d'échec demeure élevé, une transmission sur cinq échouant avant six ans. Souvent, l'insuffisance des ressources financières est tenue pour responsable notamment du fait des processus d'endettement pour le financement du rachat, avec la création d'une dette senior dans une holding créée à cet effet (Boissin, 2007). Néanmoins, «des facteurs non financiers jouant un rôle important dans la réussite ou l'échec» sont également à prendre en compte (OSÉO, 2005, p. 4). Pourtant, les organismes d'accompagnement semblent davantage se concentrer sur les aspects «techniques» du processus, relevant notamment des aspects juridiques et fiscaux, au détriment de certains aspects managériaux (Picard et Thévenard-Puthod, 2004). Étant donné les enjeux socioéconomiques sous-jacents, nous souhaitons consacrer ce travail au repérage et à la compréhension de facteurs non financiers susceptibles d'influer, également, sur la pérennité de l'entreprise. Ces facteurs peuvent être considérés comme relevant de différentes dimensions du management stratégique du processus de reprise d'entreprise. Plus particulièrement, nous nous attacherons à analyser le processus de formation de la stratégie qui s'opère à partir du moment où le repreneur prend ses nouvelles fonctions dans l'entreprise. Dans cette perspective, la richesse et la complexité des tâches qui sous-tendent la mise en place d'une stratégie constituent autant de sources de difficultés possibles. D'une part, arrivé depuis peu dans l'entreprise, le repreneur n'est socialement pas intégré. Il est souvent 
confronté au poids de l'héritage culturel de l'entreprise et à un contexte de résistance au changement et il ne bénéficie pas encore d'une position de leader. D'autre part, ayant une connaissance limitée de la structure de l'entreprise et de son environnement, la vision stratégique en construction du repreneur peut s'en trouver affectée et manquer de pertinence (Chaineau, 1989; Deschamps et Paturel, 2001).

Nous proposons tout d'abord de souligner le rôle clé du repreneur dans le processus de formation de la stratégie de l'entreprise rachetée, à partir de variables sociopsychologiques pour certaines et relatives au pouvoir discrétionnaire du dirigeant pour d'autres. À cette occasion, nous verrons également que les opportunités ont une fonction de catalyseur quant à l'influence du repreneur sur la formation de la stratégie de l'entreprise. Dans une deuxième partie, nous montrerons dans quelle mesure certaines interférences spécifiques à des cas de RPP peuvent venir troubler le processus de formation de la stratégie.

Pour mener ce travail, nous avons privilégié une approche globale et continue des processus étudiés. Aussi bien les travaux théoriques que les observations empiriques ont montré qu'il convenait de dépasser l'opposition stérile entre le délibéré et l'émergent (Martinet et Payaud, 2004). En effet, le délibéré et l'émergent sont à considérer comme deux éléments inextricablement liés et inhérents à tout processus stratégique, ce qui invite à se départir d'une pensée binaire au profit d'une pensée qui comprend les deux termes (Bernard-Weil,1988). Cette recherche s'articule autour d'un cas d'entreprise envisagé de manière approfondie et dans une perspective longitudinale.

\section{Le rôle du repreneur dans le processus de formation de la stratégie de l'entreprise}

Nous proposons de commencer par expliciter le rôle du repreneur dans le processus de formation de la stratégie de l'entreprise.

\subsection{Influence du repreneur sur la formation de la stratégie}

L'étude de la littérature permet d'envisager différents types de liens d'ordre sociopsychologique entre le repreneur et la formation de la stratégie. 


\subsubsection{Approche sociopsychologique des liens entre le dirigeant et la formation de la stratégie}

Le premier type de lien entre le dirigeant d'une entreprise et la formation de la stratégie de cette dernière est d'ordre psychologique. Les dirigeants, pour faire leur choix en matière d'organisation, se réfèrent à un système, conscient ou inconscient, de convictions personnelles concernant la nature humaine (Selznick, 1957). Zaleznick (1989) et Lapierre (1995) insistent également sur le rôle de la subjectivité des dirigeants. Enfin, dans la même lignée, Noël (1989) montre comment les obsessions des dirigeants sont à l'origine du comportement stratégique de leur organisation.

Un deuxième type de lien fait intervenir l'expérience du dirigeant, eu égard à son histoire et sa personnalité. En particulier, Smith (1967) propose une typologie permettant d'identifier, d'un côté, le dirigeant «artisan», qui souvent bénéficie d'une expérience limitée et n'a en général pas eu accès à une formation supérieure; et, d'un autre côté, le dirigeant «opportuniste», qui bénéficie d'une forte expérience de gestionnaire. Le premier aura tendance à rester sur son métier d'origine et à peu diversifier l'activité de l'entreprise, craignant de perdre le contrôle de la situation. Le second aura tendance à toujours aller de l'avant et à préférer le développement par la diversification.

Un troisième type de liens concerne les motivations et, par extension, le projet personnel du dirigeant. Marchesnay (1994) propose ainsi une classification des dirigeants en fonction de leurs motivations en termes de pérennité de l'entreprise, d'indépendance du capital ou d'autonomie de la décision et de croissance de l'entreprise. D'un côté figurent les entrepreneurs qui se situent dans une logique d'action essentiellement patrimoniale (pérennité, indépendance, croissance) et, de l'autre côté, ceux qui se situent dans une logique de valorisation du capital (croissance, autonomie, pérennité). Dans une perspective voisine, Saporta (1989) montre que le dirigeant est mû par des objectifs personnels et que son entreprise constitue pour lui un moyen de les réaliser.

Dans un contexte de RPP, à partir des travaux de Ray (1993), Deschamps (2000) montre que l'histoire et les aptitudes que le repreneur potentiel a pu développer donnent naissance à des besoins qui engendrent différents types de motivations repreneuriales. Cinq types de motivations repreneuriales ont ainsi été relevées par l'auteur: les «non-motivés», qui ne sont motivés par rien en particulier et pour qui la reprise constitue une étape transitoire dans leur carrière professionnelle; les «déterminés », dont la principale motivation 
est d'accéder à un poste de dirigeant - pour eux, la reprise d'entreprise est le fruit d'un projet de longue date; les «contraints», motivés par le fait de créer leur propre emploi; les «sociaux motivés », pour qui le caractère social de l'entreprise est prépondérant (possibilité de pérenniser une entreprise, de sauvegarder les emplois ou de redresser l'entreprise); les «investisseurs», pour qui la reprise est un simple investissement, qui peut parfois se transformer en défi. Si la motivation n'est pas l'unique élément qui conduit l'individu à la décision de reprendre et que des facteurs environnementaux sont en jeu (contexte économique et social, politiques d'incitation, mœurs contemporaines, réseaux personnels du repreneur, santé d'un secteur d'activité, etc.; Deschamps, 2000), il n'en reste pas moins qu'elle est un facteur déterminant qui conditionne la formation de la stratégie à venir de l'entreprise. En effet, les «investisseurs» ou les «déterminés» auront en général une forte propension à développer l'entreprise à travers des processus de croissance interne ou de croissance externe, alors que les «contraints» ou les «non-motivés» auront tendance à éventuellement redresser et seulement maintenir l'entreprise dans le périmètre d'activité en place au moment du rachat.

Enfin, Boissin et al. (2009) étudient quatre cas d'entreprise en croissance à partir d'une grille de lecture des typologies d'entrepreneurs. Ils concluent que les profils de dirigeant impactent le type de rythme de croissance qui sous-tend la trajectoire stratégique de l'entreprise. Ces auteurs invitent alors à considérer la croissance comme une réponse à certaines attentes du dirigeant. Alors que la croissance représente, pour l'entreprise, une finalité, s'agissant du dirigeant, elle constitue plutôt un moyen.

Ainsi, la personne du dirigeant - repreneur dans notre cas - joue un rôle central dans le processus de formation de la stratégie de l'entreprise. Dans cette perspective, nous proposons d'analyser avec quel type de regard les liens que nous venons d'évoquer sont appréhendés par les différentes parties prenantes de l'entreprise, par certains experts ou par les chercheurs en sciences de gestion.

\subsubsection{Dirigeant et formation de la stratégie: une influence souvent redoutée}

Les liens entre la personne du dirigeant et la formation de la stratégie de l'entreprise sont généralement appréhendés à deux niveaux. Le premier suggère que la stratégie personnelle du dirigeant se concrétise, de manière souvent imperceptible, par des processus émergents participant à la formation de la stratégie (Mintzberg, 1990). À un deuxième niveau, les relations entre le dirigeant et la formation de la stratégie de l'entreprise sont abordées en 
termes de gouvernement d'entreprise, selon une perspective souvent négative qui appelle à bien délimiter l'espace discrétionnaire du dirigeant (Charreaux, 1997; Boissin, Guieu et Wirtz, 2001). En dépit de ces deux constatations, nous suggérons de considérer que la stratégie personnelle du dirigeant peut se concrétiser délibérément et positivement dans le processus de formation de la stratégie de l'entreprise. Le facteur «stratégie personnelle du dirigeant» est ainsi à considérer au même titre que des éléments relevant de l'environnement externe ou des ressources de l'entreprise. Par exemple, ne serait-ce que si le dirigeant est dans une stratégie «d'enracinement interne» ou de «carrière externe ( (Charreaux, 1996), il est clair que les répercussions sur la stratégie de l'entreprise risquent d'être radicalement différentes. Dans une réflexion sur l'évolution sociologique, Touraine (2007) réhabilite l'individu en tant qu'acteur central de la vie sociale. En effet, tout individu souhaite être «créateur de lui-même», réclamant «le droit [...] de faire les choix de sa propre vie», et à la recherche d'une stratégie personnelle. Dans ce cadre, chaque individu doit parvenir, à force d'habileté, à combiner et à hiérarchiser diverses logiques de manière originale. Ainsi, tout dirigeant devrait explicitement être autorisé - voire encouragé - à se poser les questions suivantes: quelle est ma stratégie personnelle? Et, en conséquence, quelles orientations stratégiques choisir pour l'entreprise? Enfin, notons que même dans les cas où les structures de gouvernement d'entreprises sont contraignantes, un dirigeant conserve toujours une marge de manœuvre lui permettant de poursuivre ses objectifs personnels (Boissin, Guieu et Wirtz, 2001). Même en présence de mécanismes de gouvernement d'entreprise, le dirigeant conserve une latitude au niveau de ses choix stratégiques (Charreaux, 1996). À partir de là, il est illusoire de penser pouvoir neutraliser les intérêts privés du dirigeant et il est sans doute préférable de favoriser le développement d'une relation sincère entre la personne du dirigeant et les différentes parties concernées, dans un esprit de coopération et de confiance.

Ainsi, nous proposons de réintégrer explicitement et sans visée négative, la variable «stratégie personnelle» du dirigeant dans le processus de formation de la stratégie de l'entreprise. Néanmoins, cet élément ne rend pas compte à lui tout seul de la formation de la stratégie d'une entreprise. En effet, en tant que dirigeant, le repreneur est amené à façonner la stratégie de son entreprise, entre stratégie personnelle et contexte organisationnel. Dans ce cadre, l'opportunité joue un rôle central, permettant à l'agir ordinaire de s'imposer comme stratégie: «la conjonction d'une activité ordinaire couplée à un moment particulier de l'organisation [...] permet de construire une opportunité susceptible d'être alors saisie dans une décision » (Mounoud et de La Ville, 2006, p. 95). 


\subsubsection{L'opportunité: quand l'agir ordinaire s'impose comme stratégie}

Dans cette partie, l'opportunité est envisagée comme un élément central jouant le rôle de connecteur entre la pensée et l'action. En effet, les éléments à partir desquels nous venons de montrer l'influence du dirigeant sur la formation de la stratégie de l'entreprise sont des éléments qui participent à l'agir ordinaire. Néanmoins, cet agir ne peut s'imposer comme stratégie que grâce aux opportunités qui auront été construites par le dirigeant, puis concrétisées sous forme de décisions. Considérons tout d'abord l'exemple donné par Mintzberg (1990) à propos du potier en train de tourner son argile, pour en faire une sculpture. Soudain, l'argile entre en contact avec un ébauchoir et une forme ronde commence à apparaître: c'est alors que le potier a une nouvelle idée, celle de réaliser un vase cylindrique plutôt qu'une sculpture. L'hypothèse traditionnelle selon laquelle la pensée est indépendante de l'action se trouve ainsi rejetée. Au contraire, la connexion reliant la pensée à l'action devient «la clef de l'art du potier» (Mintzberg, 1990, p. 53). Mais, plus précisément, en quoi consiste cette connexion? Dans l'exemple du potier, un événement a surgi: l'argile est entrée en contact avec un ébauchoir et une forme ronde a commencé à apparaître. Puis, le potier a choisi de transformer cet événement en une opportunité de réaliser un vase cylindrique plutôt qu'une sculpture. C'est sa perception subjective de la situation qui lui a permis de faire ce choix et d'amorcer le processus de transformation d'événement en opportunité. En effet, l'opportunité n'est pas naturelle, préexistante à l'activité du potier. Elle est le fruit d'une démarche de construction (Ngijol et Chabaud, 2004). Dans cette perspective, interpréter un événement comme une opportunité ou comme une menace relève d'une opération hautement subjective (Avenier, 1988). Les opportunités sont subjectives dans la mesure où elles dépendent du degré d'ambiguïté de l'environnement et de la capacité des acteurs sociaux à développer les modèles mentaux nécessaires pour les interpréter et pour les définir comme opportunités (Companys et McMullen, 2007). Pour exister, les opportunités ont besoin d'être définies et énactées par les individus (Weick, 1979). Par conséquent, une opportunité n'est pas découverte, elle est plutôt perçue, construite ou énactée par un individu en particulier. Ce n'est qu'ex-post, c'est-à-dire une fois exploitée, que l'opportunité acquiert un caractère objectif (Ngijol et Chabaud, 2004). La clé de l'art du potier et, par analogie, celle du stratège (Mintzberg, 1990), consisterait à savoir transformer certains événements en opportunités. Dans ce cadre, l'opportunité est à considérer comme un connecteur entre la pensée et l'action et, en particulier, entre la stratégie personnelle du dirigeant et la formation de la stratégie de l'entreprise. 


\section{FIGURE 1}

Influence du repreneur dans le processus de formation de la stratégie de l'organisation

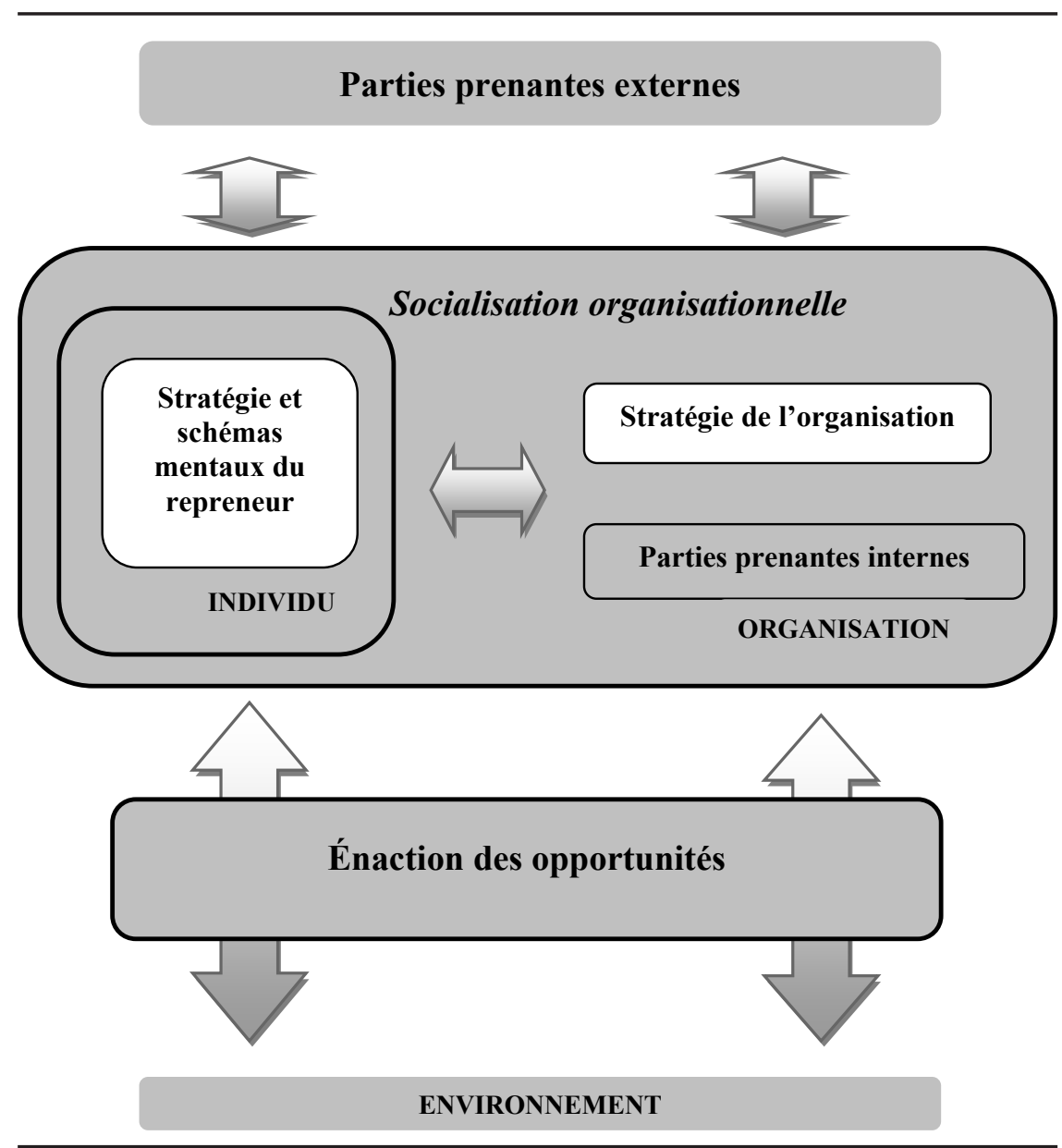

À partir de l'étude de cas CIC ORIO, nous proposons maintenant de mettre en perspective les différents éléments théoriques que nous venons d'évoquer. 


\subsection{Le cas de l'entreprise CIC ORIO}

Après une présentation de la méthodologie à partir de laquelle l'étude de cas a été construite, le terrain d'expérience sera présenté, puis nous mettrons en perspective les propositions théoriques effectuées précédemment, avec les informations recueillies sur le terrain.

L'étude de cas menée pour ce travail n'a pas pour objectif de tester des hypothèses, mais plutôt d'éclairer un certain nombre de propositions construites dans le cadre d'une démarche exploratoire. Étant donné la nature du phénomène que nous étudions - complexe et dynamique-, ce type d'étude est particulièrement approprié (Eisenhardt, 1989; Yin, 1994) car il offre la possibilité de saisir les situations dans leur ensemble et de procéder à un examen approfondi des processus organisationnels en jeu.

Les données ont été collectées sur une période de deux ans allant de décembre 2005 à décembre 2007. Nous avons réalisé 15 entretiens semidirectifs enregistrés, d'une durée comprise entre 45 minutes et 2 heures, auprès du dirigeant de l'entreprise (huit), de son épouse (un) et de certains salariés (six). Précisons que les quatre salariés avec lesquels nous avons mené des entretiens ont été choisis de manière aléatoire, essentiellement en fonction des disponibilités de chacun. En outre, le dirigeant de l'entreprise désirait explicitement s'engager avec nous dans une démarche réflexive, avec le souci de prendre du recul par rapport à son quotidien (Denis, Langley et Rouleau,2007). Cette collaboration active a facilité notre travail. Enfin, nous avons complété les entretiens par l'analyse détaillée des plans d'affaires et par une phase d'observation (une conférence donnée par le repreneur).

La société de Chaudronnerie industrielle de Claix (CIC) a été fondée en 1987 à Jarrie (Isère) par monsieur Romero, chaudronnier de formation. Société anonyme à responsabilité limitée (SARL) au capital de quelques milliers d'euros, cette petite chaudronnerie industrielle s'est développée jusqu'en 2000 essentiellement autour de trois sites de la région de Grenoble. Son activité s'est peu à peu étendue à la construction métallique, à la serrurerie et à l'entretien général d'usine. Cette entreprise fait preuve d'une excellente réputation technique auprès de ses clients. En 2005, la société compte sept salariés, dont l'épouse du gérant, un plombier chauffagiste et cinq chaudronniers serruriers. En raison de l'anticipation de leur départ en retraite, les propriétaires de l'entreprise arrêtent progressivement d'investir et décident de vendre leur entreprise. Sans aucune démarche commerciale active, l'entreprise reste malgré tout surchargée de travail.

Le protocole a été signé le 28 octobre 2005. Le repreneur et leader du projet, Olivier Six, est gérant majoritaire. Le rachat de l'entreprise a été 
financé à $60 \%$ par un prêt bancaire et à $40 \%$ par les capitaux propres d'un holding créé le $1^{\text {er }}$ décembre 2005 (ORIO), dont Olivier Six est majoritaire. Ses deux associés détiennent respectivement $12 \%$ et $2,6 \%$ des parts de la holding. Le 2 janvier 2006, l'entreprise CIC a été définitivement cédée à ORIO. Le rachat de l'entreprise CIC (SARL) s'est effectué à travers l'acquisition de titres.

Nous montrerons successivement l'influence du repreneur sur la formation de la stratégie de son entreprise puis nous mettrons en lumière le rôle joué par l'opportunité dans ce processus d'influence.

Avant d'envisager les différents types de liens qui se sont construits entre la personne du repreneur et la formation de la stratégie de l'entreprise CIC ORIO, nous détaillons certains points concernant l'histoire, les aptitudes, les besoins et les motivations repreneuriales d'Olivier Six.

Le repreneur de CIC est issu d'une famille qu'on ne peut pas vraiment caractériser d'entrepreneuriale: son père est cadre dirigeant chez Philips Semiconducteur et sa mère, après une formation d'infirmière, est devenue mère au foyer. Ingénieur des Arts et Métiers de Bordeaux, diplômé en 1997, il a d'abord passé six ans dans l'armée de terre (management de terrain de 30 mécaniciens puis responsable technique des ateliers de maintenance). Étouffé par les lourdeurs administratives, il a quitté son poste pour être embauché chez Ponticelli Frères, dans le secteur de la maintenance industrielle, avec l'espoir de plus d'autonomie et la volonté de trouver un côté entrepreneurial plus prononcé. Il a travaillé dans cette société entre 2003 et 2005 et y a connu une évolution professionnelle intéressante mais pas suffisante à son goût, relativement déçu du manque de reconnaissance dont lui témoignait l'entreprise et de la patience dont on lui demandait de faire preuve (au vu de son âge) avant d'évoluer. Les principales motivations d'Olivier Six pour la reprise, nées à partir des besoins liés à ses antécédents, sont les suivantes:

L'autonomie et la liberté d'entreprendre: avoir la possibilité de créer et de développer ses projets avec d'autres contraintes que leur faisabilité, leur rentabilité et la nécessité de convaincre les partenaires et salariés. Le fait de gagner sa vie en fonction de ses résultats: pouvoir recevoir le fruit de son travail et de ses résultats (bons ou mauvais), sans avoir à dépendre d'une grille de salaires ou à attendre d'avoir l'âge nécessaire pour accéder à tel ou tel poste. (Entretien du 15 décembre 2005.)

Au niveau de l'environnement, le repreneur a été très encouragé par la santé du secteur d'activité dans lequel il souhaitait reprendre une entreprise, le milieu des services industriels lourds: il nous confie que «la demande est largement supérieure à l'offre» (externalisation de ce type d'activité par les entreprises, départs en retraite, etc.) et que le marché est «très porteur» 
(entretien du 15 décembre 2005). En termes de motivation repreneuriale, il semblerait qu'Olivier Six fasse partie du groupe des repreneurs «déterminés», ayant d'importants projets de développement pour l'entreprise rachetée. Néanmoins, cette suggestion mériterait d'être éventuellement confirmée à l'issue d'une étude plus approfondie.

Analysons à présent les interactions qui se sont effectivement produites entre la stratégie personnelle d'Olivier Six et la formation de la stratégie de l'entreprise. Pour mieux mettre en valeur les liens entre le dirigeant et la formation de la stratégie de l'entreprise, nous procéderons par comparaison entre les périodes ante et post reprise.

Quelques mois avant d'être transmise, l'entreprise CIC est dirigée par monsieur Romero, dont la stratégie personnelle s'articule autour d'un départ à la retraite proche:il ne souhaite plus s'investir de manière inconditionnelle dans son travail. En revanche, Olivier Six, entrepreneur dynamique, indépendant et ambitieux, soutenu dans ses choix par son épouse, se situe au début d'une carrière prometteuse.

Des liens significatifs apparaissent entre les stratégies personnelles de chacun des dirigeants et le processus de formation de la stratégie, sur les périodes correspondant respectivement à chaque dirigeant. Durant les dernières années, sous la direction de monsieur Romero, la stratégie de l'entreprise stagne, sans démarche commerciale active. Ce dirigeant «artisan» ne souhaite pas absorber une demande croissante mais fait le nécessaire pour que son entreprise reste une cible attractive à la vente. En revanche, Olivier Six a des projets de développement ambitieux pour l'entreprise. Dirigeant «opportuniste», il aborde la reprise avec deux nouvelles orientations stratégiques importantes en termes de développement et de croissance de l'entreprise: d'une part, reprendre et développer l'activité existante et, d'autre part, lancer une nouvelle activité, la mécanique industrielle. Sur le plan du chiffre d'affaires, on constate une très forte augmentation entre 2005 et $2006(+123 \%)$. Après une année de croissance interne importante (le nombre d'employés a été multiplié par deux en moins de six mois, passant de 8 à 16), il rachète, en décembre 2006, une entreprise de chaudronnerie puis, en avril 2007, une serrurerie de la région. Nous établissons ainsi un premier lien entre le projet de carrière du dirigeant et la croissance de l'entreprise.

Le deuxième type de liens que nous avons observé s'articule autour de la personnalité et de la culture du dirigeant. Avec monsieur Romero, nous sommes en présence d'un dirigeant très paternaliste, qui encadre de près le travail exécuté par ses salariés. Il se rend indispensable et contrôle tout en permanence. Le dirigeant étant très sollicité par la gestion du quotidien et ses salariés ne bénéficiant que de peu d'autonomie, la stratégie de l'entreprise 
est stable. Au contraire, Olivier Six voit les choses différemment: «Ce qui m'amuse, c'est de construire, de bâtir les choses. Gérer, peaufiner, améliorer, ce n'est pas ce qui me passionne beaucoup. $\mathrm{Si}$ au bout d'un an la situation n'a pas évolué, ce n'est pas drôle. [...] Ce qui me plaît précisément, c'est de creuser le tunnel, d'étayer comme il faut, puis de mettre en place la structure qui fait que ça marche derrière» (entretien du 15 décembre 2005).

Ces aspirations se répercutent en termes de dynamisme par rapport à la formation de la stratégie de l'entreprise. Mais un tel foisonnement d'idées et de projets ne peut pas fonctionner avec le même type d'organisation du travail que sous monsieur Romero. C'est pourquoi Olivier Six insiste sur l'importance de s'entourer de collaborateurs compétents et responsables, auxquels il faut déléguer. C'est l'un de ses associés qui s'est occupé de lancer la nouvelle activité, tandis que le second a pris en charge sur un plan technique l'activité existante et son développement. D'autre part, Olivier Six travaille à améliorer la valorisation des salariés en vue d'augmenter leur motivation et leur efficacité. Comme toile de fond, ce dirigeant considère que «ce qui est important, c'est l'harmonie entre les différents acteurs (employés, clients, fournisseurs et dirigeants). Pour cela, le rôle du leader est important, car il fédère les gens qui sont autour de lui, mais il faut surtout que, de manière harmonieuse, les gens fassent ce qu'ils ont envie de faire» (entretien du 29 décembre 2005).

Ainsi, nous sommes confrontés à deux personnalités et deux cultures très différentes: monsieur Romero aime encadrer et contrôler, alors qu'Olivier Six préfère construire puis déléguer pour la gestion. Les méthodes d'organisation et de travail proposées par les deux dirigeants tranchent donc radicalement l'une avec l'autre, les unes poussant plutôt l'entreprise à la stagnation, les autres favorisant l'entretien d'une dynamique de croissance. Ces liens théoriques que nous avons établis ne sont pourtant pas suffisants pour éclairer l'ensemble de la dynamique dans laquelle s'insère le repreneur lorsqu'il façonne la stratégie au sein de l'entreprise. Certes, le repreneur présente certaines dispositions et intentions qui favorisent telle ou telle stratégie pour l'entreprise. Néanmoins,

[...] la recherche a été découragée par la manière particulière de concevoir le concept de stratégie. Dans la littérature, la stratégie a tout le temps été définie en termes d'intentions, donnant les grandes lignes pour le futur - essentiellement en termes de plans. [...] Mais concevoir la stratégie en termes d'intentions signifie restreindre la recherche à l'étude des perceptions de ce que ceux qui sont supposés faire de la stratégie ont l'intention de faire. Et ce type de recherche - à partir d'intentions dénuées de comportement - est simplement peu intéressant ou productif (Mintzberg et Waters, 1982, p. 465, traduction libre). 
Ces auteurs préconisent de considérer davantage le caractère énacté de la stratégie, notamment en travaillant sur les liens entre stratégie délibérée et stratégie émergente. Essentiellement en termes d'intentions, nous avons montré que stratégie personnelle du dirigeant et formation de la stratégie de l'entreprise sont liées. Mais à travers quelles interactions ces liens se concrétisent-ils?

Considérons tout d'abord les propos tenus par le dirigeant actuel de CIC ORIO, Olivier Six:

Je pense que l'important, c'est de savoir saisir des opportunités. Je n'ai pas forcément d'objectifs, j'ai plutôt une philosophie et une vision des choses. Est-ce qu'on veut croître? Vers quoi? Aller à l'international? etc. Je dirais, des grandes lignes. Et après, ce qui est intéressant pour moi, c'est de saisir les opportunités qui se présentent. C'est beaucoup plus facile d'étudier un dossier, de dire oui ou non, plutôt que de dire «mon objectif, c'est d'être là dans tant de temps, et pour y aller je vais mettre une énergie folle», alors que se dire «je veux aller vers là-bas, suivre le chemin avec ses sinuosités, faire comme la rivière qui passe entre les montagnes et qui va de toute façon toujours vers la mer», c'est une philosophie qui est beaucoup moins fatigante [...] (Entretien du 15 décembre 2005.)

Cet extrait d'entretien permet d'appréhender une partie des modèles mentaux sous l'influence desquels se situe le repreneur de l'entreprise. La nouvelle organisation et le changement d'encadrement nécessitent alors plus d'autonomie de la part de ces derniers. Mais Olivier Six est confiant: «Quand j'ai présenté le projet il y a un mois, j'ai bien senti dans les yeux des gars une flamme d'intérêt pour s'investir. [...] Cela leur a plu, je pense qu'ils sont attirés par ce côté responsabilisation et un peu plus d'autonomie » (Entretien du 2 février 2006). Trois mois après la reprise, voilà ce qu'il en est:

On a presque six mois d'avance par rapport au développement prévu. Le chiffre d'affaires du premier trimestre est supérieur de $33 \%$. Au niveau du personnel aussi, on embauche beaucoup plus vite que prévu. [...] Tout le monde court, il y a une forte demande du côté des clients. Il faut prendre beaucoup de décisions en même temps [...] En plus de cela, il ne faut pas démotiver l'équipe, il faut l'aider à absorber les jeunes. [...] On est plutôt en mesure de refuser du travail, alors qu'on ne fait pas de commercial. Le souci actuel, c'est la productivité [...] On consomme plus d'heures qu'avant sur une même affaire, 10 \% en plus, notamment parce qu'on a deux jeunes sans expérience et un ancien qui n'a pas travaillé depuis cinq ans. Il faudrait qu'on arrive à augmenter le personnel sans augmenter l'encadrement terrain. Au niveau des chefs d'équipe, ce n'est pas encore optimal. (Entretien du 7 avril 2006.)

À la même période, il ressort des entretiens avec les employés qu'ils sont mécontents, voire incompétents, face à la nouvelle organisation du travail (ils ne souhaitent pas endosser la responsabilité d'éventuelles erreurs). 
Néanmoins, malgré ces tensions, Olivier Six, au lieu de freiner brusquement la croissance de l'activité, prend le risque de tout mettre en œuvre pour l'absorber et la soutenir: une nouvelle personne est embauchée pour pourvoir à l'échelon d'encadrement manquant et un plan d'intéressement financier basé sur trois critères (la sécurité, la qualité et la productivité) est mis en place, équivalent à un treizième mois, soit un fait encore rare au sein d'une PME métallurgique. À partir de sa stratégie personnelle, ce dirigeant a donc su transformer divers événements initialement non prévus (croissance trop rapide de l'activité et de l'entreprise) en opportunités pour développer l'entreprise sur le plan stratégique.

Nous constatons une démarche similaire en ce qui concerne le recrutement. Pour ce repreneur, «vouloir à tout prix recruter, c'est catastrophique. [...] C'est très cher en termes de publication d'annonces et très chronophage. Ce que je fais maintenant, c'est que j'attends et puis quand les gens passent, même si on n'en a pas besoin, on les embauche, quand ils sont bien, parce qu'on en aura besoin plus tard» (entretien du 18 janvier 2007).

Enfin, les rachats successifs des deux entreprises de chaudronnerie et de serrurerie se sont eux aussi déroulés à partir d'événements transformés en opportunités. À l'époque, la chaudronnerie concernée sous-traitait du travail à CIC ORIO. Tenu au courant par un client de la faillite de cette chaudronnerie, Olivier Six contacta la société et les négociations en vue du rachat s'enclenchèrent. Une fois le rachat effectué, CIC ORIO intégra des locaux plus adaptés et plus spacieux, bénéficiant d'un meilleur équipement industriel, plus approprié à ses activités. L'effectif global de l'entreprise est alors passé de 15 à 25 salariés.

Puis, l'activité fleurissante de CIC ORIO conduisit quatre mois plus tard au rachat d'une serrurerie. Dans le but de vendre son entreprise, l'ancien patron de cette serrurerie était venu rencontrer Olivier Six, à partir de la liste des chaudronniers de la région. Après une rapide étude et la rencontre d'un des principaux clients, CIC ORIO a racheté le fonds de commerce.

Les deux nouvelles entités ont été complètement refondues dans la nouvelle organisation, autour de métiers transversaux, avec un dirigeant, cinq chargés d'affaires, trois secrétaires administratives et comptables et 25 chefs d'équipe et compagnons. Aujourd'hui, CIC ORIO est devenu un acteur incontournable du service au monde industriel. Sa réputation grandissante et sa clientèle lui confèrent une image de marque favorable à son développement. La flexibilité de la production et de la structure organisationnelle en vue de rendre un service maximum aux clients («le service, c'est notre raison d'être » - entretien du 4 décembre 2007) est un atout majeur de cette entreprise. 
Avec le concept d'opportunité, nous avons ainsi contribué à éclairer la manière dont des liens peuvent concrètement se construire entre le repreneur et la formation de la stratégie de l'entreprise, entre délibéré et émergent. Néanmoins, certaines interférences - propres à des cas de RPP - peuvent venir influencer ce processus de formation de la stratégie.

\section{Le repreneur : entre vision stratégique et socialisation organisationnelle}

Dans le cadre d'une RPP, deux interférences risquent principalement de se produire dans le processus de formation de la stratégie, en lien avec le processus de socialisation organisationnelle du repreneur, d'une part, et en lien avec la vision stratégique, d'autre part.

\subsection{Principales sources d'interférences dans la formation de la stratégie en contexte de RPP}

Par sa récente entrée dans l'entreprise, le repreneur n'a encore acquis ni leadership ni reconnaissance de la part de ses employés et pourtant, il a besoin que l'organisation entière participe au processus de formation de la stratégie en cours et à venir. Lorsqu'il entre dans l'entreprise, le repreneur se voit attribuer «son nouveau rôle de propriétaire-dirigeant» (Deschamps, 2000), mais des difficultés peuvent alors survenir liées au fait que le repreneur devient «le dirigeant d'un personnel qu'il n'a pas recruté, dans une entreprise qu'il n'a pas fondée, qui a fonctionné avant lui, sans lui à sa tête » (Deschamps et Paturel, 2001). C'est alors qu'il doit franchir les différentes étapes du processus de socialisation organisationnelle afin d'acquérir les valeurs, les comportements et les connaissances nécessaires pour tenir son rôle dans l'organisation et en être considéré comme un véritable membre (Feldman, 1976; Louis, 1980).

Boussaguet (2005) montre qu'on peut même observer un processus de socialisation organisationnelle particulier au repreneur, «processus par lequel le repreneur parvient à faire la "transition" pour assumer son rôle de leader et se faire accepter en tant que tel par les salariés en place» (Boussaguet, 2006, p. 3). En effet, au moment où il entre dans l'entreprise, si le repreneur possède un statut formel, il lui reste une légitimité à conquérir. De nombreuses étapes sont donc à franchir qui conditionnent le processus de formation de la stratégie. Plus précisément, le repreneur se trouve confronté à des difficultés en termes de leadership, de culture d'entreprise et de résistances de la part des salariés. 
Vis-à-vis de ses salariés, le repreneur n'est pas encore reconnu comme un leader. Parmi les trois éléments qui composent le leadership (la vision, la confiance et l'adhésion; Aubert, 1991, cité par Boussaguet, 2005), s'il peut arriver que le repreneur en possède un, la vision, il ne peut en général compter ni sur la confiance, ni sur l'adhésion de ses salariés.

Le repreneur doit également composer avec l'héritage culturel que lui laisse le cédant et qui n'est pas forcément compatible avec les évolutions stratégiques qu'il projette de mener. La difficulté pour le repreneur est de prendre conscience du poids de cet héritage culturel. Plus précisément, il peut se produire un véritable choc entre la culture du repreneur et celle de l'entreprise qu'il rachète (Deschamps, 2000). Certains soutiennent même que les organisations peuvent devenir captives de leur propre héritage culturel: il faut alors rompre l'inertie stratégique accumulée qui a imprégné la culture de l'entreprise (Balogun et Hope Hailey, 2004).

La résistance au changement constitue une autre des difficultés auxquelles le repreneur se voit confronté, tout changement étant à l'origine de résistances et de crises (Crozier et Friedberg, 1977).

Le repreneur, peinant à obtenir une légitimité au sein de l'entreprise qu'il vient de reprendre, est donc confronté à une série d'obstacles freinant le processus de formation de la stratégie à venir. La tâche est d'autant plus ardue qu'il est soumis à une tension permanente, écartelé entre deux pôles opposés:

Un repreneur, soucieux de ne pas perturber l'ancienne organisation peut, en se conformant trop, ne pas apporter une véritable valeur ajoutée et a contrario, un repreneur pressé de marquer et de transformer l'organisation qu'il rachète, peut en perturber le fonctionnement. Ce qui contribuera au succès ou à l'échec du début de la reprise est [...] le bon dosage du degré de socialisation organisationnelle au moment de l'entrée (d'Andria, 2006, p. 7).

Le degré de socialisation organisationnelle du repreneur s'avère donc être déterminant dans le déroulement de la partie opérationnelle du processus stratégique.

Un autre élément peut venir interférer dans le processus de formation de la stratégie: le repreneur, par sa récente arrivée dans l'entreprise et son manque d'expérience, peut éprouver des difficultés à se constituer une vision stratégique adéquate. 
Le concept de vision stratégique, dont l'origine se trouve dans les travaux de Hamel et Prahalad (1989)1, est défini avec différentes nuances selon les auteurs. Deux points communs apparaissent néanmoins.

Tout d'abord, les auteurs se rejoignent pour dire que la vision stratégique est un critère essentiel de performance des organisations (Van de Ven, Huston et Schroeder,1984; Filion,1991; Verstraete,1999). À partir d'une vision stratégique basée sur une utilisation pertinente des ressources de l'entreprise, l'objectif est de faire évoluer la logique du champ concurrentiel (Aubret, Gilbert et Pigeyre,2005), de poser les règles du jeu de demain et de construire un chemin plutôt que d'en suivre un qui existe déjà (Verstraete, 2001).

D'autre part, dans toutes les définitions proposées, le concept de vision stratégique «semble toujours désigner une produit cognitif mobilisateur, en évolution et constituant un système référentiel - un schème - d'où émerge une certaine orientation» (Cossette, 2004, p. 108).

Ainsi, par nature, la vision stratégique d'un dirigeant contient une importante dimension cognitive (Verstraete,2001) et est fortement liée à la personne du dirigeant, à son histoire, à sa manière de percevoir les événements qui se produisent. Or, faute d'une clairvoyance et d'une capacité d'adaptation suffisantes, le repreneur peut avoir de la difficulté à établir une vision stratégique adéquate, étape pourtant décisive en matière de pérennité de l'entreprise. N'ayant pas encore en main toutes les clés d'analyse et de compréhension nécessaires, il lui est très difficile d'acquérir aussi rapidement qu'il le faudrait un bon niveau d'acuité stratégique, c'est-à-dire un degré de finesse suffisant du point de vue de la vision stratégique (Revolon et Delecroix, 1998).

Finalement, il apparaît que le processus de socialisation organisationnelle et le niveau d'acuité stratégique du repreneur sont susceptibles d'interférer dans le processus de formation de la stratégie. Voyons comment ces éléments ont impacté le processus de formation de la stratégie de l'entreprise CIC ORIO.

\subsection{Mise en lumière des interférences dans la formation de la stratégie de CIC ORIO}

Dans la lignée des réflexions théoriques menées ci-avant, nous montrerons successivement la manière dont des interférences dans la formation de la stratégie, en lien avec le processus de socialisation organisationnelle et la vision stratégique du repreneur, se sont concrétisées dans le cas CIC ORIO.

1. En effet, ces auteurs «ont développé le concept d'intention stratégique comme un état futur de l'organisation basé sur les aspirations du dirigeant et de ses membres afin de les motiver» (Boissin et al., 2009). 
Étant donné qu'il a quitté son précédent travail juste avant le moment de reprendre l'entreprise, Olivier Six ne disposait que de peu de temps pour préparer son intégration dans l'entreprise : il n'a suivi aucune formation particulière ou préparation psychologique. Il n'a rencontré les salariés qu'une seule fois avant son entrée dans l'entreprise: c'était au milieu du mois de décembre, une quinzaine de jours avant la reprise. Quant à l'ancien cédant, peu enclin à communiquer, il n'a pas du tout contribué à préparer le terrain. Lors de son entrée dans l'entreprise, Olivier Six a le sentiment d'être tiraillé dans deux directions différentes: s'adapter ou s'imposer. Celui-ci s'attribuait ainsi deux grands rôles:

Rassurer [...] au niveau de la pérennité de l'entreprise. D'autre part, il faut que les salariés aient confiance dans les personnes de la nouvelle équipe, qu'ils ne les perçoivent pas comme des re-créateurs, qu'ils aient vraiment l'impression que le changement va se faire en douceur, qu'ils ne vont pas être ni trop perturbés, ni biaisés. [...] C'est la première phase de consolidation. Motiver, créer un dynamisme et donner envie de faire plus. (Entretien du 29 décembre 2005.)

Lors de cette phase, il a reçu les employés un à un afin de mieux percevoir leurs attentes. La confrontation à la réalité du terrain et les premières décisions ont également contribué à le faire avancer dans le processus de socialisation organisationnelle. Il améliore notamment sa connaissance des salariés: même si certains sont de bonne volonté, ils ne sont finalement pas aussi autonomes et responsables que prévu. Quant aux salariés, ils semblent apprécier les qualités humaines et relationnelles d'Olivier Six - «jeune», «sympa» (salarié 1, entretien du 30 mai 2006) -, et «l'ambiance super» de l'entreprise (salarié 2, entretien du 30 mai 2006)). Par contre, d'un point de vue technique, le repreneur et ses associés ont plus de mal à se faire reconnaître, surtout en comparaison de l'ancien dirigeant, qui était très compétent: «Monsieur Romero a beaucoup de poids dans l'entreprise, il est d'un grand secours pour tout le monde. [...] Après son départ, certains vont souffrir. Cédric [un des associés] est plus intellectuel que manuel: il n'est pas fait pour l'encadrement du travail de terrain. [...] Il a moins d'expérience que monsieur Romero» (salarié 1, entretien du 30 mai 2006).

Reprenons les trois points qui composent le leadership (la vision, la confiance et l'adhésion des salariés) et voyons ce qu'il en est pour le repreneur de CIC ORIO.

- La vision. Comme nous l'avons vu, Olivier Six a élaboré très tôt sa vision stratégique. Il sait parfaitement où il souhaite emmener l'entreprise. Ce point ne semble donc pas poser de problème.

- La confiance. Concernant l'avenir de l'entreprise, il semble que les qualités relationnelles d'Olivier Six («Olivier est beaucoup 
plus ouvert, il discute avec tout le monde», salarié 4, entretien du 2 février 2006) ainsi que sa jeunesse et son dynamisme l'aient aidé à obtenir la confiance de ses employés. Par contre, sur un plan technique, les employés ne sont pas du tout rassurés: «J'ai toujours confiance en mon ancien patron. Pour l'instant, le nouveau, on ne s'en occupe pas. [...] C'est lui le patron, mais quand on a un conseil, on ne va pas le voir. Un chef d'atelier va arriver bientôt, pour remplacer Romero» (salarié 3, entretien du 2 février 2006).

- L'adhésion. Si les employés sont globalement satisfaits de la marge d'autonomie que prévoient de leur laisser les projets du repreneur, certains ne sont pas très contents du stress qui va avec: «l'équipe des trois patrons veut aller trop vite. [...] On n'était pas boostés comme ça avant. Sur chaque chantier, ils veulent aller trop vite. [...] On ne fait pas du meilleur boulot, mais on speed beaucoup plus » (salarié 2, entretien du 2 mars 2006). Quant au plan d'intéressement financier: «ça vaut rien leur truc, ils sont les seuls à pouvoir vérifier les bénéfices. C'est très compliqué. Je suis le seul à avoir fait une remarque pendant la réunion. Les autres ont rouspété après » (salarié 2 , entretien du 2 mars 2006). Un autre, plus modéré, mentionne seulement des problèmes au niveau de l'organisation du travail et des changements trop fréquents d'équipes.

Globalement, on ne peut pas dire qu'Olivier Six soit déjà leader dans l'entreprise même s'il est plutôt sur la bonne voie pour le devenir. Il lui reste surtout à obtenir la confiance de ses employés sur un plan technique.

L'héritage culturel de CIC est lourd, et les méthodes d'organisation et de travail proposées par Olivier Six tranchent radicalement avec ce qui était pratiqué avec le cédant. À présent, tout est basé sur l'autonomie, la responsabilité, la confiance alors qu'avant, le dirigeant contrôlait tout en permanence et préparait systématiquement le travail de ses employés, simples exécutants: «il est de l'ancienne école», mais «c'était un père pour nous! » (salarié 1, entretien du 2 mars 2006). Les changements insufflés par le repreneur demandent donc de gros efforts d'adaptation de la part des ouvriers, sachant que la fermeture de leur entreprise ne leur cause pas d'inquiétude: «je n'ai pas eu peur de perdre mon emploi. Tout le monde embauche. Rien ne me retient dans cette boîte» (salarié 4, entretien du 2 février 2006).

Nous avons déjà observé plusieurs manifestations de résistance au changement dans les paragraphes précédents consacrés au terrain. Néanmoins, il est important de souligner qu'au début de la reprise, l'entreprise comptait huit personnes alors qu'au moment où cette étude a été réalisée, elle en 
comptait 16. Cela a donc permis d'apporter du sang neuf dans l'entreprise, si bien que seule la moitié du personnel est réellement concernée par les problèmes de résistance au changement. Pour l'autre moitié, ce type de difficultés ne se pose pas. On ressent d'ailleurs nettement la différence au niveau des entretiens réalisés : un ouvrier déjà présent dans l'entreprise avant la reprise est beaucoup plus vindicatif qu'un ouvrier embauché après.

Ainsi, le niveau de socialisation organisationnelle du repreneur est venu influencer le processus de formation de la stratégie de CIC ORIO. Voyons ce qu'il en est concernant l'acuité de la vision stratégique d'Olivier Six.

Concernant CIC, le repreneur a élaboré sa vision stratégique (ou du moins un début) avant d'entrer dans l'entreprise. Travaillant auparavant dans le même secteur d'activité que celui de l'entreprise reprise, il disposait de certaines connaissances lui permettant de tracer assez rapidement des perspectives pour l'avenir. Pour lui, la reprise de CIC est composée de «deux grandes parties: d'une part, la reprise et le développement de l'activité existante et, d'autre part, le lancement d'une nouvelle activité : la mécanique industrielle» (entretien du 29 décembre 2005). Plus concrètement, Olivier Six considère que «la qualité du service offert continuera d'être une valeur importante de l'entreprise. Les changements consisteront à améliorer la valorisation des salariés (quelqu'un de valorisé est plus motivé et donc plus productif et efficace dans le travail réalisé), ainsi que la politique de commercialisation et la réactivité. Ce qui est important, c'est l'harmonie entre les différents acteurs (employés, clients, fournisseurs et dirigeants). Pour cela, le rôle du leader est important, car il fédère les gens qui sont autour de lui, mais il faut surtout que, de manière harmonieuse, les gens fassent ce qu'ils ont envie de faire» (entretien du 29 décembre 2005).

Par sa bonne connaissance du secteur d'activité et par sa philosophie générale, Olivier Six dispose d'un bon niveau d'acuité stratégique. En témoigne le développement florissant de l'entreprise, y compris à l'heure d'aujourd'hui. Plusieurs dizaines de salariés ont été embauchés depuis cette étude. Néanmoins, un défaut peut être souligné en termes d'acuité stratégique. En effet, Olivier Six, en se basant sur une mauvaise estimation des ressources de l'entreprise, a voulu trop rapidement passer à l'action: «On pensait que les gars seraient plus autonomes. Du coup, il manque un échelon d'encadrement» (Olivier Six, entretien du 30 mai 2006).

Comme nous l'avons vu précédemment avec d'autres extraits d'interviews, cela a créé des tensions au sein du personnel qui, pendant un moment, a peiné à s'adapter. L'impatience de ce repreneur aurait donc pu être la cause de sérieux ennuis, si ce dernier n'avait pas eu les réactions appropriées dont 
il a su finalement faire preuve pour réajuster un certain nombre d'éléments, et s'il n'avait pas rapidement embauché plusieurs employés. Les anciens se sont ainsi trouvés noyés dans la masse, leurs revendications ne devenant plus que minoritaires.

À partir d'une revue de la littérature et de l'étude de cas, nous avons donc mis en évidence l'interférence, plus ou moins forte, de deux facteurs dans le processus de formation de la stratégie, propres à une situation de RPP: le niveau de socialisation organisationnelle du repreneur et la construction d'une vision stratégique adéquate. En outre, s'agissant du deuxième de ces facteurs, l'interférence peut se trouver amoindrie lorsque le repreneur provient du même secteur d'activité.

\section{Conclusion}

L'objectif de ce travail était d'améliorer la compréhension du processus de RPP, plus particulièrement en termes de formation de la stratégie. Notons que les enseignements obtenus à partir de cette recherche n'ont pas vocation à être généralisés, mais sont plutôt destinés à fournir aux praticiens des points de repères qui leurs seront utiles, dans la perspective de l'élaboration de savoirs génériques (Avenier et Gialdini, 2009).

La revue de la littérature et l'étude de cas nous ont permis de mettre en lumière certaines relations étroites entre la personne du repreneur, la vision stratégique, le processus de socialisation organisationnelle et la formation de la stratégie de l'entreprise acquise.

La personnalité du repreneur, son système de valeurs, son expérience et ses motivations sont autant d'éléments qui interviennent dans la perception que cet individu se construit d'une situation, dans les opportunités qu'il énacte et dans les actions et décisions qui s'en suivent et impactent la formation de la stratégie de l'entreprise. Parallèlement, deux types de difficultés propres à une situation de RPP peuvent se produire: il s'agit de certaines interférences liées au processus de socialisation organisationnelle et à la construction d'une vision stratégique par le repreneur.

Tous ces éléments sont inextricablement liés les uns aux autres et influent simultanément sur le processus de formation de la stratégie.

Enfin, étant donné que les facteurs qui interviennent dans le processus de formation de la stratégie de l'entreprise récemment acquise dépendent étroitement de la personne du repreneur, il apparaît fortement nécessaire d'analyser attentivement s'il existe une réelle adéquation entre les projets du repreneur et les possibilités qu'offre l'entreprise en termes de stratégie 
possible pour le futur. En effet, toute entreprise ne peut pas absorber tout type de stratégie. C'est pourquoi une étude détaillée des projets du repreneur (liés à sa personnalité, son système de valeurs, son expérience, ses motivations, etc.) doit être effectuée, afin de vérifier l'adéquation (Kotter, 1982) entre la personne du repreneur et la fonction de direction qu'il souhaite occuper au sein d'une entreprise spécifique.

Concernant les perspectives futures de cette recherche, il semblerait pertinent, étant donné la croissance connue par l'entreprise CIC ORIO depuis son rachat jusqu'à aujourd'hui, de s'interroger pour savoir si nous avons affaire à un «serial repreneur» ou si les acquisitions qui ont suivi le premier rachat relèvent du processus de croissance externe de l'entreprise originelle. Au regard du discours du repreneur, qui laisse transparaître un vif intérêt pour de potentielles opportunités de croissance, y compris celles qui supposeraient une diversification des activités, nous pencherions plutôt pour la première configuration.

\section{Bibliographie}

Aubert, N. (1991), Management, aspects humains et organisationnels, Paris, Presses universitaires de France, Fondamental.

Aubret, J., P. Gilbert et F. Pigeyre (2005), Management des compétences, Paris, Dunod.

AVENIER, M.-J. (1988), Le pilotage stratégique de l'entreprise, Paris, Presses du CNRS.

AVENIER, M.-J. et L. GiALDINI (2009), «Capturer de la connaissance pratique pour élaborer des savoirs académiques: un cadre méthodologique pour étudier la "fabrique de la stratégie" ", Cahier de recherche CERAG, n 2009-02, p. 1-26.

Balogun, J.et V. Hope Hailey (2004), Exploring Strategic Change, Londres, Prentice Hall.

BERNARD-WeIL, E. (1988), Précis de systémique ago-antagonisme, Limonest, Interdisciplinaire.

BoISsin, J.-P. (2007), «Trouver de nouvelles structures de transmission d'entreprises », dans A. Fayolle (dir.), L'Art d'entreprendre, Paris, Village Mondial - Pearson, p. 234-243.

Boissin, J.-P., M.-C. Chalus-Sauvannet, B. Deschamps et S. Geindre (2009), «Profils de chercheurs primo-entrepreneurs et stratégies de croissance de la jeune entreprise innovante», Revue internationale $P M E$, vol. 22, $\mathrm{n}^{\circ} 2$.

Boissin, J.-P., G. GUIEU et P. WIRTZ (2001), «Les fusions internationales - une lecture à travers la théorie institutionnelle», dans A.-C. Martinet et R.-A. Thiétart (dir.), Stratégies: actualités et futurs de la recherche, Paris, Vuibert, p. 249-272. 
Boussaguet, S. (2005), L'entrée dans l'entreprise du repreneur: un processus de socialisation repreneuriale, Thèse de doctorat en sciences de gestion, Université de Montpellier.

Boussaguet, S. (2006), «Réussir son entrée dans l'entreprise: le processus de socialisation du nouveau dirigeant », Actes des Ires Journées Georges-Doriot.

Chaineau, A. (1989), La transmission d'entreprises: enjeux et perspectives, Paris, Presses universitaires de France.

Charreaux, G. (1996), «Pour une véritable théorie de la latitude managériale et du gouvernement des entreprises », Revue française de gestion, vol. 111, p. 50-64.

Charreaux, G. (1997), "Vers une théorie du gouvernement des entreprises», dans G. Charreaux (dir.), Le gouvernement des entreprises, Paris, Economica, coll. «Recherche en gestion», p. 421-469.

COMPANYS, Y. et J. MCMULLEN (2007), «Strategic entrepreneurs at work : the nature, discovery, and exploitation of entrepreneurial opportunities », Small Business Economics, vol. 28, p. 301-322.

CossetTe, P. (2004), L'organisation: une perspective cognitiviste, Québec, Les Presses de l'Université Laval.

Crozier, M. et E. Friedberg (1977), L'acteur et le système, Paris, Seuil.

D'ANDRIA, A. (2006), «Réalités et difficultés de l'entrée à la socialisation organisationnelle du repreneur. Le cas d'un établissement hôtelier», Les Ires Journées Georges-Doriot.

Denis J.-L., A. LANGLEY et L. RouleAU (2007), « Strategizing in pluralistic contexts : rethinking theoretical frames», Human Relations, vol. 60, nº 1, p. 179-215.

DESCHAMPS, B. (2000), Le processus de reprise d'entreprise par les entrepreneurs personnes physiques, Thèse de doctorat en sciences de gestion, Université de Grenoble.

DESCHAMPS, B. et R. PATUREL (2001), Reprendre une entreprise... saine ou en difficulté, Paris, Dunod, coll. «Entrepreneurs».

EISENHARDT, K.M. (1989), «Building theories from case study research», Academy of Management Review, vol. 14, n 4, p. 532-550.

Feldman, D.C. (1976), «A contingency theory of socialization», Administrative Science Quarterly, vol. 21, p. 433-452.

FiLION, L.J. (1991), Vision et relations: clefs du succès de l'entrepreneur, Montréal, Éditions de l'Entrepreneur.

HAmel, G. et C.K. Prahalad (1989), «Strategic intent», Harvard Business Review, p. $148-161$.

KOTTER, J.P. (1982), «General managers are not generalists », Organizational Dynamics, printemps.

LAPIERRE, L. (1995), «La subjectivité, l'autorité et la direction, leçon et contre-leçon inaugurales », Cahier des leçons inaugurales, HEC Montréal, février. 
LOUIS, M.R. (1980), «Surprise and sensemaking: what newcomers experience in entering unfamiliar organization settings », Administrative Science Quarterly, vol. 25 , p. 226-251.

MARTINeT, A.C. et M.A. PAYAUD (2004), «Stratégisation de l'organisation et écologisation de la stratégie: les savoirs et relations du middle manager stratège ordinaire», $A S A C$, Québec.

MARCHESNAY, M. (1994), Management stratégique, Paris, Eyrolles.

MinTzBeRG, H. (1990), Le management: voyage au centre des organisations, Paris, Éditions d'Organisation.

MinTZBERG, H. et J. WATERS (1982), «Tracking strategy in an entrepreneurial firm », Academy of Management Journal, vol. 25, n 3, p. 465-499.

MounOud, E. et V.-I. DE LA VILLE (2006), «La créativité de l'agir ordinaire : éléments pour une approche énactive de la stratégie», dans D. Golsorkhi (dir.), La fabrique de la stratégie, une perspective multidimensionnelle, Institut Vital-Roux, Paris, Vuibert, p. 109-128.

NGiJOL, J. et D. Chabaud (2004), «La reconnaissance des opportunités de marché par l'entrepreneur: vers une perspective constructiviste», Cahier de recherche $D M S P, \mathrm{n}^{\circ} 326$.

NOËL, A. (1989), «Strategic cores and magnificent obsessions: discovering strategy formation through daily activities of CEOs», Strategic Management Journal, été.

OSÉO (2005), La transmission des petites et moyennes entreprises en France, <http:// oseov5.novactive.fr/votre_projet/transmission/s_informer/regard_d_oseo_sur_ le_marche/etude_oseo_transmission_2005>.

PICARD, C. et C.THÉVENARD-PUTHOD (2004), «La reprise de l'entreprise artisanale: spécificités du processus et conditions de sa réussite», Revue internationale $P M E$, vol. 17, no 2, p. 94-121.

RAY, D.M. (1993), «Understanding the entrepreneur: entrepreneurial attributes, experience and skills», Entrepreneurship and Regional Development, vol. 5, p. 345-357.

REVOlOn, F. et C. DeleCROIX (1998), «L'acuité stratégique de l'entrepreneur », Actes de l'AIMS, Chatenay-Malabry.

SAPORTA, B. (1989), «Stratégies des PME», dans P. Joffre et Y. Simon (dir.), Encyclopédie de gestion, Paris, Economica, p. 2729-2754.

SElZnICK, P. (1957), Leadership in Administration: A Sociological Interpretation, New York, Harper and Row.

SMITH, N.R. (1967), «The entrepreneur and his firm: the relationship between type of man and type of company», Occasional Papers, Bureau of Business and Economic Research, Michigan State University, vol. 109.

Touraine, A. (2007), Penser autrement, Paris, Fayard.

VAn DE Ven, A., R. Huston et D.M. Schroeder (1984), «Designing new business start-ups », Journal of Management, vol. 10, $\mathrm{n}^{\circ} 1$. 
VERSTRAETE, T. (1999), Entrepreneuriat: connaître l'entrepreneur, comprendre ses actes, Paris, L'Harmattan, coll. «Économie et innovation».

VERSTRAETE,T.(2001), «Entrepreneuriat et stratégie entrepreneuriale: quels modèles pour la recherche et l'action?", Les Cahiers de la recherche, le CLAREE.

WeICK, K.E. (1979), The Social Psychology of Organizing, Reading, Mass., AddisonWesley.

YIN, R.K. (1994), Case Study Research: Design and Methods, Londres, Sage.

ZALEZNIK, A. (1989), The Managerial Mystique: Restoring Leadership in Business, New York, Harper and Row. 